\title{
Cumplimiento integral del programa de higiene de manos basado en el modelo del consejo de salubridad general (México)
}

\section{Comprehensive compliance with the hand hygiene program based on the model of the general health council (Mexico)}

\author{
Jesús Eduardo Romo Martínez, ${ }^{*}$ Sandra Guadalupe Rivas Cobos, ${ }^{*}$ Ilse Marelyn Portillo García, ${ }^{*}$ \\ Rodolfo Chávez Chávez, ${ }^{\ddagger}$ Rogelio Reyes Soto, ${ }^{\S}$ Elia Yvette Anaid Ortiz Rivas, IIvonne Loera Estradall
}

\section{Resumen}

Introducción: Las infecciones asociadas con la atención a la salud (IAAS) son una amenaza potencial en la atención sanitaria; la manera más efectiva de prevenirlas es el cumplimiento de la higiene de manos (HM) junto con otros factores esenciales, los cuales fueron propuestos por el Consejo de Salubridad General y fueron expuestos en la implementación del Programa integral de higiene de manos (PIHM). Objetivo: Describir y analizar los resultados de la implementación del PIHM en el Hospital Ángeles Chihuahua (HAC). Material y métodos: Estudio observacional y longitudinal en el que se evaluó de forma cuantitativa y mensual los resultados de las verificaciones del PIHM. Resultados: La adherencia global (año 2018) del programa resultó en un puntaje de 92\%. Discusión: El cumplimiento de la evaluación del PIHM durante este año, sin duda, proyecta la maduración de los procesos hacia un mejoramiento continuo.

Palabras clave: Higiene de manos, implementación.
* Unidad de Vigilancia Epidemiológica Hospitalaria.

₹ Dirección General.

§ Dirección Médica y Presidente del Comité para la Detección y Control de las Infecciones Nosocomiales.

I| Dirección de Planeación y Calidad.

Hospital Ángeles Chihuahua. Chihuahua, Chihuahua.

Correspondencia:

Dr. Jesús Eduardo Romo Martínez

Correo electrónico: jesusromo.epidemio@gmail.com

Aceptado: 12-12-2019.

www.medigraphic.com/actamedica

\section{Abstract}

Introduction: Health care associated infections (HCAl) are a potential threat in health care and the most effective way to prevent them is through compliance with hand hygiene $(\mathrm{HH})$ coupled with other essential factors proposed by the Council of Health General Health (CSG in Mexico) proposing the implementation an Integral Program of Hand Hygiene. Objective: Describe and analyze the results of the implementation of Integral Program of $\mathrm{HH}$ in the Hospital Angeles Chihuahua (HAC). Material and methods: Longitudinal observational study in which the results of the Integral Program of $\mathrm{HH}$ verifications are evaluated on a monthly basis. Results: Global adherence (year 2018) of the program resulted in 92\%. Discussion: The fulfillment of the evaluation of the Integral Program of $\mathrm{HH}$ during this year, undoubtedly projects the maturation of processes towards continuous improvement.

Keywords: Hand hygiene, implementation.

\section{INTRODUCCIÓN}

Las infecciones asociadas con la atención a la salud (IAAS) son una amenaza potencial, tanto para el paciente y su familia como para los sistemas de salud propiamente dichos, por las repercusiones que éstas conllevan, tales como: morbilidad, letalidad sobre la estancia hospitalaria, costos de atención, problemas legales, etc. ${ }^{1-6}$

La manera más efectiva de prevenir las IAAS es el cumplimiento de la higiene de manos (HM) en los cinco momentos y con la técnica adecuada, tal como lo dispone la Organización Mundial de la Salud (OMS). Sólo con cumplir con la HM garantizaría contener el riesgo de IAAS; no obstante existen además otros factores indirectos tales como el aseguramiento y abasto de insumos necesarios; la capacitación profesional formal del personal clínico 
en cuanto a los momentos y técnica de HM se refiere; la educación y orientación a los pacientes, su familia y visitantes; garantizar la calidad del agua y, evidentemente, el monitoreo y apego a las buenas prácticas. ${ }^{7}$

En México, la prevención y control de infecciones, así como su vigilancia epidemiológica son ámbitos que están lo suficientemente apoyados por bases normativas, lineamientos y procesos estandarizados. ${ }^{7-10}$ Estas bases, lineamientos y procesos, actualmente, se consideran dentro de los sistemas críticos que se norman dentro del Consejo de Salubridad General (CSG) ${ }^{7}$ para la certificación de establecimientos. Esta certificación propone una serie de estándares bien estructurados con los mínimos necesarios para su cumplimiento y, a su vez, describe una de las Metas Internacionales: la Meta Internacional para la Seguridad del Paciente núm. 5, que compete además a la línea de acción esencial en el Programa integral de higiene de manos (PIHM).

En el Hospital Ángeles Chihuahua se ha instalado un programa sistematizado de prevención y control de infecciones que está apegado estrictamente a los estándares vigentes del CSG, ${ }^{11}$ e inclusive sus resultados provenientes de experiencias ya se han divulgado y han mostrado buenos resultados. ${ }^{12}$

El propósito de este artículo es difundir la adherencia de la implementación del PIHM en Hospital Ángeles Chihuahua durante el año 2018.

\section{MATERIAL Y MÉTODOS}

Se trata de un estudio observacional del tipo longitudinal, en el que se evaluaron los cinco componentes del PIHM propuestos por el CSG, bajo la metodología del proceso de certificación de establecimientos de atención médica.
Se contemplaron las siguientes líneas de acción:

a) Monitorización de la calidad del agua (cumplimiento de la NOM correspondiente a los parámetros de cloración establecidos).

b) Abasto de insumos necesarios.

c) Educación a pacientes y familiares (efectividad de la orientación en la importancia de la higiene de manos al realizar la encuesta).

d) Capacitación del profesional clínico en higiene de manos.

e) Cumplimiento de la higiene de manos (observada mediante un estudio de sombra).

Para cada una de las líneas de acción, se realizó una lista de verificación digital (formularios) que contenían los puntos mínimos y necesarios para dar cumplimiento cabal a las acciones. Estas evaluaciones se realizaron de manera mensual, excepto la del cumplimiento de higiene de manos, la cual se llevó a cabo con visorias aleatorias continuas. De estas evaluaciones, se estimaron los porcentuales del cumplimiento y se analizaron mediante un mosaico que contempla la rendición de cuentas para cada línea mensual, así como la evaluación integral mensual del PIHM mediante una línea de tiempo que mantuvo su respectiva línea de tendencia, la cual permitió entender la madurez del proceso a lo largo del año.

\section{RESULTADOS}

El desempeño porcentual global del PIHM en el Hospital Ángeles Chihuahua, durante 2018, demostró una adherencia favorable para las cinco líneas de acción propuestas para ser evaluadas (Tabla 1). La calidad del agua y el abasto

\begin{tabular}{|c|c|c|c|c|c|c|c|c|c|c|c|c|}
\hline Línea de acción/mes & Ene & Feb & Mar & $\mathrm{Abr}$ & May & Jun & Jul & Ago & Sep & Oct & Nov & Dic \\
\hline $\begin{array}{l}\text { a) Monitorización de la } \\
\text { calidad del agua }\end{array}$ & 89.0 & 100.0 & 100.0 & 100.0 & 100.0 & 100.0 & 100.0 & 100.0 & 100.0 & 100.0 & 100.0 & 100.0 \\
\hline $\begin{array}{l}\text { b) Abasto de insumos } \\
\text { necesarios }\end{array}$ & 99.0 & 99.1 & 99.8 & 98.8 & 98.2 & 99.3 & 99.8 & 100.0 & 100.0 & 100.0 & 99.5 & 100.0 \\
\hline $\begin{array}{l}\text { c) Educación a pacientes } \\
\text { y familiares }\end{array}$ & 95.0 & 99.4 & 100.0 & 100.0 & 100.0 & 100.0 & 99.9 & 99.9 & 99.8 & 99.8 & 99.8 & 98.0 \\
\hline $\begin{array}{l}\text { d) Capacitación al profe- } \\
\text { sional }(\mathrm{PCl})\end{array}$ & 97.0 & 80.0 & 80.0 & 90.0 & 80.0 & 91.8 & 95.1 & 95.1 & 93.0 & 90.0 & 89.2 & 91.0 \\
\hline $\begin{array}{l}\text { e) Cumplimiento con la } \\
\text { higiene de manos**}\end{array}$ & 75.0 & 63.2 & 63.6 & 68.3 & 81.0 & 82.7 & 66.9 & 75.2 & 74.7 & 70.1 & 92.0 & 87.0 \\
\hline $\begin{array}{l}\text { Evaluación integral del } \\
\text { PIHM }\end{array}$ & 91.0 & 88.3 & 88.7 & 91.4 & 91.8 & 94.8 & 92.3 & 94.0 & 93.5 & 92.0 & 96.1 & 95.2 \\
\hline
\end{tabular}




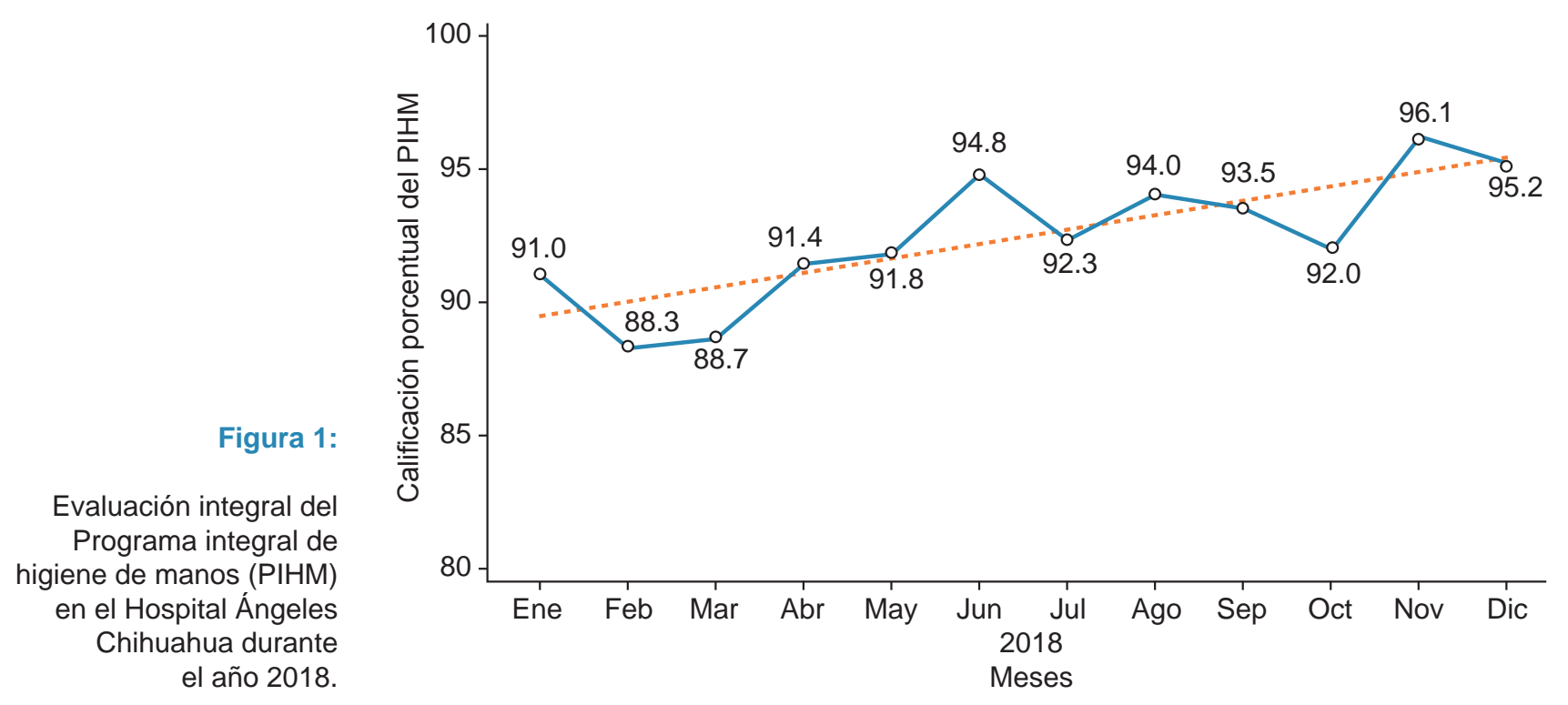

de insumos se mantuvieron con resultados inmejorables a lo largo de todos los meses.

Para el caso de los puntos referentes a la capacitación profesional y educación a pacientes y familiares, los resultados, aunque con variantes, también fueron destacables por encima de $90 \%$. Sin embargo, en el punto respectivo al cumplimiento de la higiene de manos, valorado mediante un estudio de sombra, hubo cambios irregulares entre meses, con un mínimo en los 60 puntos y hasta un máximo en los 92 puntos porcentuales.

El desempeño porcentual global del PIHM durante el 2018 resultó con un promedio general de 92\%. Durante el año se mantuvo con un comportamiento hacia el alza, con un mínimo observado en febrero de 88.3 porcentual y un máximo en noviembre de 96.1 porcentual (Figura 1).

\section{DISCUSIÓN}

Las fortalezas de esta evaluación destacan tanto como el gran trabajo en equipo y la disposición de proyectar avances hacia la mejora. En esta evaluación, las situaciones imprescindibles tales como garantizar el insumo para la higiene de manos, así como la calidad de un abastecimiento de agua segura se cumplieron a lo largo de los meses de forma casi automatizada; aquellos rubros de incumplimiento respondieron a los sustentos de las mediciones en medios físicos como bitácoras (libros), pero sin vulnerar los procesos. Para el caso de la capacitación de personal, así como la orientación a los pacientes y a sus familiares, aunque los resultados fueron muy altos, nos encontramos con el natural fenómeno de rotación de personas (personal laboral, en formación y los mismos visitantes de pacientes); por ende, fue difícil asegurar $100 \%$ en tiempo real de estos rubros. No obstante, el cumplimiento del rubro de higiene de manos observado mediante los estudios de sombra sí evidenció resultados a considerar por debajo de los noventa porcentuales; sin embargo, éstos son comparables positivamente con estadísticas nacionales.

Como limitación de este estudio pudiéramos mencionar la necesidad de continuar midiendo de forma proactiva estas líneas de acción y, convenientemente, comparar entre los años, situación que se empezó a evaluar en 2018. Las mediciones que fueron cualitativas $(\mathrm{S} i / \mathrm{No})$ en razón de evaluar cumplimientos, por ejemplo, para la medición de la calidad del agua, fueron Ilevadas a cabo en una lista de verificación, la cual tuvo un total de 12 aspectos a evaluar; de ello ponderamos directamente el porcentual de cumplimientos "Sí". Quizá valdría la pena dar puntajes de No = 0, Sí = 1, 2, 3, etc., dependiendo de la importancia del rubro a evaluar.

Sin duda, los resultados de la evaluación del PIHM durante este año proyectan la maduración de procesos hacia la mejora continua.

Esperamos que este material sirva de referencia para dimensionar y autoevaluar este proceso indispensable de todos los establecimientos de atención médica con miras a sujetarse a los estándares de certificación. Se debe tomar en cuenta que esta evaluación puede ser aplicada por varios evaluadores enfocados en los procesos.

\section{REFERENCIAS}

1. Acosta SI. Manual de control de infecciones y epidemiología hospitalaria. Washington, D.C.: Organización Panamericana de la Salud; 2011. pp. 3-11. 
2. Romo J, Ortega C, Arriaga J. Estudio de prevalencia de infecciones nosocomiales en un hospital de tercer nivel de atención. Enf Inf Microbiol. 2014; 34 (1): 6-12.

3. Romo-Martínez JE, González-Barrera JA, Guillén-Rincón MI, Herrera-Solís ME, Vicenteño-Muriño A. Letalidad por infecciones nosocomiales en un hospital general regional, desde un enfoque epidemiológico. Salud Pública Méx. 2015; 57 (1): 2-3.

4. Malagón G, Moreno CA. Infecciones hospitalarias. 3a ed. Bogotá, Colombia: Editorial Médica Panamericana; 2010. pp. 14-24.

5. Ponce de León S, Rangel MS, Elías JI, Romero C, Huertas M. Infecciones nosocomiales: tendencias seculares de un programa de control en México. Salud Pública Mex. 1999; 41 (Supl. 1): S511.

6. Malagón G, Pontón J, Reynales J. Gerencia hospitalaria, para una administración efectiva. 4a ed. México: Editorial Médica Panamericana; 2012.

7. Consejo de Salubridad General, Modelo de Seguridad del Paciente del Consejo de Salubridad General. Estándares para implementar el modelo en hospitales 2015. 3a edición. México: 2017.

8. Dirección General de Epidemiología. Manual de procedimientos estandarizados para la vigilancia epidemiológica hospitalaria. México: 2016.

9. Diario Oficial de la Federación. Norma Oficial Mexicana NOM-045SSA2-2005, Para la vigilancia epidemiológica, prevención y control de las infecciones nosocomiales. México, 2009.

10. Diario Oficial de la Federación. Norma Oficial Mexicana NOM017-SSA2-2012, Para la vigilancia epidemiológica. México, 2013.

11. Romo MJE, Chávez CR, Reyes SR et al. Generalidades de un sistema de prevención y control de infecciones asociadas a la atención a la salud. Enf Infec Microbiol. 2018; 38 (1): 24-26.

12. Romo MJE, Chávez CR, Reyes SR et al. Mejora de la calidad y seguridad del paciente en el sistema crítico de prevención y control de infecciones en el Hospital Ángeles Chihuahua. Acta Med. 2019; 17 (1): 86-88. 